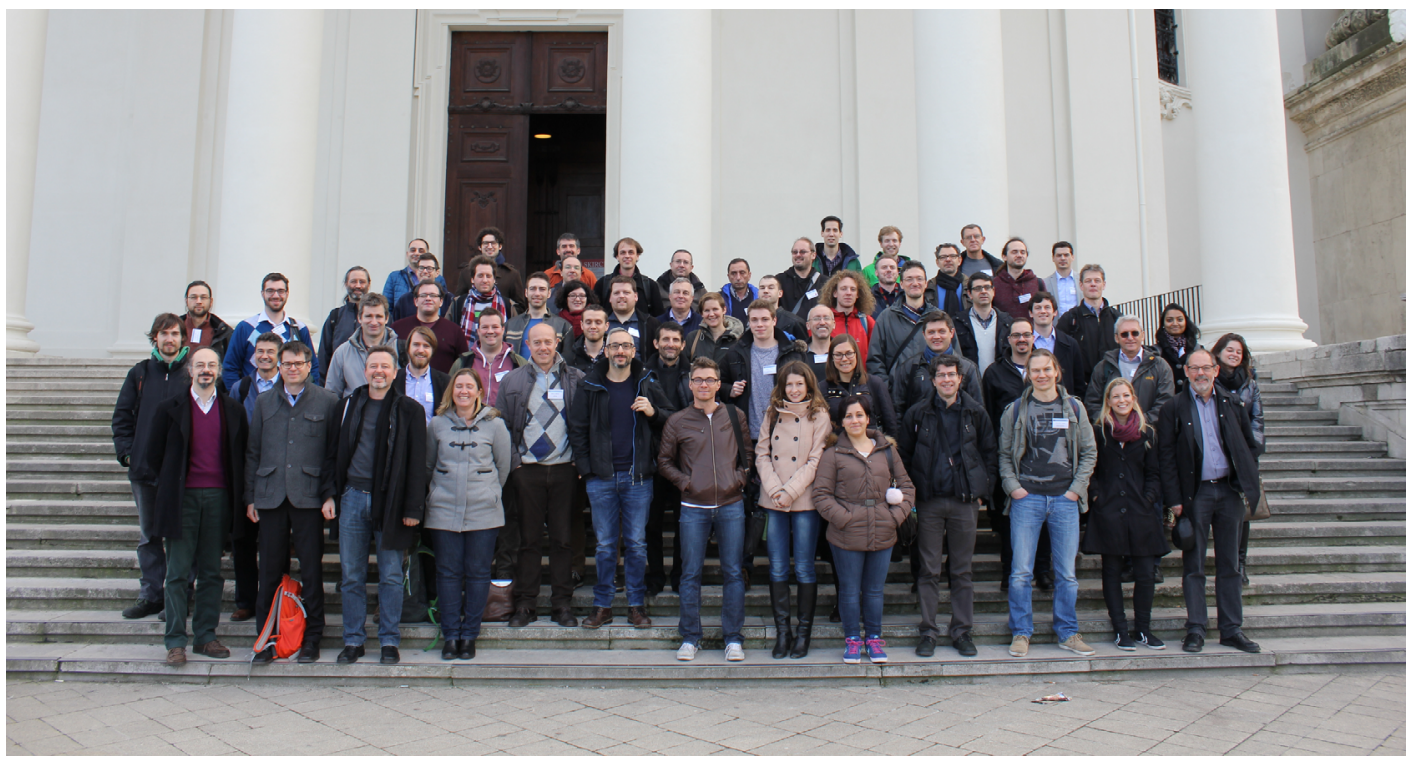

These are the conference proceedings of the workshop "Connecting The Dots 2016" that was held in Vienna, Austria, from February 22 to February 24, 2016, see:

http://ctd2016.hephy.at

The workshop concentrated on track reconstruction and other problems in pattern recognition in sparsely sampled data. Such problems are abundant in particle physics, and, consequently, most contributions were rooted in high energy physics experiments and primarily revolved around the reconstruction of particle tracks and jets. Nevertheless, the workshop was intended to be cross-disciplinary; similar problems in other fields were identified and discussed, and contributions from astrophysics, astronomy, or antiproton decelerator facilities also appeared on the agenda.

Talks were by invitation only; in total, 74 participants attended the workshop, 39 talks were given, 16 of which are represented in these conference proceedings. A "Young Scientists Forum" gave our younger colleagues the opportunity to claim the stage in front of an international audience and present their own, personal work. Within this workshop, a meeting of the "Common Track Reconstruction Forum" was held, aiming at an efficient exchange of concepts, algorithms and, ultimately, software:

\title{
http: //hepsoftwarefoundation.org/workinggroups/2016/02/02/tracking.html
}

The workshop has been received positively by our various scientific communities. It is therefore our goal to establish a "Connecting the Dots" workshop series. Following the inaugural workshop last year at LBNL Berkeley and this year's workshop in Vienna, the series will be continued in March of next year at LAL Orsay, France. Detailed information about the workshop will appear online in due course.

The local organizers:

Rudi Frühwirth, Erica Brondolin, Bernadette Kolbinger, Wolfgang Waltenberger 